\title{
Lycopene Improves Bone Quality and Regulates AGE/RAGE/NF- KB Signaling Pathway in High-Fat Diet-Induced Obese Mice
}

\author{
Bingke Xia, ${ }^{1}$ Ruyuan Zhu, ${ }^{1}$ Hao Zhang, ${ }^{1}$ Beibei Chen, ${ }^{1}$ Yage Liu, ${ }^{1}$ Xuan Dai, ${ }^{1}$ \\ Zimengwei Ye, ${ }^{1}$ Dandan Zhao ${ }^{D},{ }^{1}$ Fangfang Mo, ${ }^{1}$ Sihua Gao ${ }^{1},{ }^{1}$ Xiang-Dong Wang, \\ Dieter Bromme, ${ }^{3}$ Lili Wang, ${ }^{4}$ Xinxiang Wang, ${ }^{5}$ and Dongwei Zhang $\mathbb{D}^{1}$ \\ ${ }^{1}$ Diabetes Research Center, Traditional Chinese Medicine School, Beijing University of Chinese Medicine, Beijing 100029, China \\ ${ }^{2}$ Jean Mayer USDA Human Nutrition Research Center on Aging at Tufts University, Boston, MA 02111, USA \\ ${ }^{3}$ Department of Oral Biological \& Medical Sciences, Faculty of Dentistry, The University of British Columbia, Vancouver, BC, \\ Canada V6T $1 Z 3$ \\ ${ }^{4}$ Department of TCM Pharmacology, Chinese Material Medica School, Beijing University of Chinese Medicine, \\ Beijing 102488, China \\ ${ }^{5}$ The Scientific Research Center, Dongfang Hospital, Beijing University of Chinese Medicine, Beijing 100078, China
}

Correspondence should be addressed to Dongwei Zhang; dongwei1006@gmail.com

Received 16 June 2021; Revised 9 January 2022; Accepted 28 January 2022; Published 17 February 2022

Academic Editor: Dragan Hrnčić

Copyright (c) 2022 Bingke Xia et al. This is an open access article distributed under the Creative Commons Attribution License, which permits unrestricted use, distribution, and reproduction in any medium, provided the original work is properly cited.

Objective. This study was aimed at examining the effects of lycopene on bone metabolism in high-fat diet (HFD)- induced obese mice and to identify the potential underlying mechanisms. Methods. Mice were fed a HFD for 12 weeks and then continue with or without lycopene intervention $(15 \mathrm{mg} / \mathrm{kg}$ ) for additional 10 weeks. The effects of lycopene on blood glucose and lipid metabolism, as well as serum levels of total antioxidant capacity (T-AOC), superoxide dismutase (SOD), and malondialdehyde (MDA) were determined by biochemical assays. Bone histomorphological features and osteoclast activity were assessed by hematoxylin/eosin and tartrate-resistant acid phosphatase staining. Bone microstructure at the proximal tibial metaphysis and diaphysis was determined by microcomputed tomography. Tibial biomechanical strength and material profiles were measured by a threepoint bending assay and Fourier transform infrared spectroscopy. Protein expressions involved in the AGE/RAGE/NF- $\mathrm{KB}$ signaling pathway were determined by western blot and/or immunohistochemical staining. Results. Lycopene consumption reduced body weight gain and improved blood glucose and lipid metabolism in HFD-induced obese mice. In addition, lycopene treatment preserved bone biomechanical strength, material profiles, and microarchitecture in obese mice. Moreover, these alterations were associated with an increase in serum levels of T-AOC and SOD, and a decline in serum levels of MDA, as well as a reduction of AGEs, RAGE, cathepsin K, and p-NF-кBp65 and NF-кBp65 expressions in the femurs and tibias of obese mice. Conclusion. Lycopene may improve bone quality through its antioxidant properties, which may be linked with the regulation of the AGE/RAGE/NF- $\mathrm{B}$ signaling pathway in obese mice. These results suggest that lycopene consumption may be beneficial for the management of obesity-induced osteoporosis.

\section{Introduction}

As a metabolically active tissue, bone is regulated by systemic glucose and lipid metabolism [1]. Given the worldwide prevalence of obesity and hyperglycemia, the study of bone metabolism has gained increasing attention [2-4]. Accumulating evidence suggests that sustained dyslipidemia and hyperglyce- mia impair bone microstructure, material compositions, and biomechanical strength, leading to an increased risk of bone fractures [5-7]. However, clinical studies revealed that bone quality is mostly neglected during obesity management $[8$, 9]. In addition, current pharmacological countermeasures for obesity and osteoporosis are associated with poor adherence and adverse complications [10]. Emerging evidence suggests 
that dietary intervention may provide a new strategy for the effective management of bone and lipid metabolism [11-13].

Advanced glycation end products (AGEs), mainly derived from sustained hyperglycemia, drive oxidative stress generation and abnormal bone remodeling [14, 15]. AGEs may promote osteoclastic bone resorption and inhibit osteoblastic bone formation through the regulation of the receptor for AGEs (RAGE) [16]. In addition, AGEs compromise bone quality and increase fracture risk through altering bone material constituents [17]. Moreover, elevation of AGEs enhances the production of reactive oxygen species (ROS), which further triggers oxidative stress via RAGE. These interactions result in the activation of nuclear factor-kappa $\mathrm{B}(\mathrm{NF}-\mathrm{\kappa} \mathrm{B})$ translocation and promote subsequent cathepsin $\mathrm{K}$ (catK) expression, leading to increased bone resorption $[18,19]$. As has been shown previously, mice deficient in RAGE exhibit a decline in osteoclasts and an increase in bone mass [20]. In addition, our previous studies suggested that the inhibition of AGEs/RAGE/NF- $\mathrm{BB}$ and NADPH oxidase $4 / \mathrm{ROS} / \mathrm{NF}-\mathrm{\kappa B}$ signaling improves bone quality in diabetic and ovariectomized rats $[16,18]$. Bucala et al. also showed that the administration of an advanced glycation inhibitor to diabetic subjects helps to prevent dyslipidemia [21]. Thus, the inhibitions of AGEs and ROS generation may offer a new strategy of protection against metabolic syndrome-related derangements involving bone tissue.

Lycopene, a dietary carotenoid, mainly found in tomatoes and other red colored fruits [19], is well known for its high antioxidant potential [22]. There are accumulating evidences suggesting that lycopene consumption helps to improve bone metabolism [23-26]. We and others have demonstrated that lycopene could improve blood glucose and lipid metabolism in preclinical studies and clinical trials $[27,28]$. However, the possibility that lycopene administration may prevent bone loss in patients with glucose and lipid metabolism disorders remains unexplored. Therefore, our aim for the present study is to examine the effects of lycopene on bone quality in high-fat diet (HFD)- induced obese mice, as well as its associations with the AGE/RAGE/NF- $\mathrm{KB}$ signaling pathway.

\section{Materials and Methods}

2.1. Reagents and Antibodies. Lycopene was purchased from RuiFenSi Biotechnology Co., Ltd. (Chengdu, China). The glucose consumption assay kit was obtained from Applygen Technologies Inc. (Beijing, China). Antigen retrieval solution was bought from ShunBai Biotechnology Company (No. SBT10013; Shanghai, China). Total antioxidant capacity (T-AOC), superoxide dismutase (SOD), and malondialdehyde (MDA) kits were purchased from Nanjing Jiancheng Bioengineering Institute (Nanjing, China). Antibodies against AGEs (ab23722), RAGE (ab37647), and catK (ab19027) were from Abcam Biocompany (Cambridge, MA, USA). Antibodies against p-NF-кBp65 (WL02169) and NFкBp65 (WL01980) were from Wanlei Biotechnology (Shenyang, China). Antibodies against GAPDH (60004-1-Ig) and $\beta$-actin (66009-1-Ig) were from Proteintech Biotechnology (Wuhan, China). HRP-conjugated secondary antibodies were procured from Proteintech Biotechnology. All other reagents excluding those previously identified were from Sinopharm Reagents Co. Ltd (Beijing, China).

2.2. Animals. Male Institute of Cancer Research (ICR) mice weighing about $20 \pm 2 \mathrm{~g}$ were bought from Beijing SiBeiFu Animal Technology Co. Ltd. (Beijing, China) and housed in the specific-pathogen-free (SPF) animal facility with a constant temperature $\left(22^{\circ} \mathrm{C} \pm 2{ }^{\circ} \mathrm{C}\right)$ and humidity $(55 \% \pm 5 \%)$ and a 12 -h light/dark cycle at the Beijing University of Chinese Medicine (BUCM). All mice were supplied with regular chow and drinking water ad libitum. All the animal procedures were approved by the BUCM Animal Care Committee, Beijing, China.

2.3. Diet-Induced Obesity and Lycopene Administration. After acclimation for 1 week, the ICR mice were fed a HFD (45\% fat, Rodent Diet D12032; Research Diet, Jiangshu, China) for 12 consecutive weeks. The HFD-fed mice that met the following requirements were enrolled in the subsequent experiments [29]: (1) body weight gain greater than $20 \%$ and (2) a fasting glucose level over $7.8 \mathrm{mM}$. The eligible mice were randomly divided into three groups and treated for additional 10 weeks as shown in Table 1 . In addition, 9 mice fed a regular chow diet were used as normal controls.

During the experiment, the body weight of the mice was measured once a week. At the end of treatment after anesthesia, body weight and length were measured. Blood was harvested from the heart for further analysis. Meanwhile, the bilateral tibias and femurs were removed from the animal bodies. The samples were then collected according to the requirements for further experiments.

2.4. Glucose Tolerance Tests. An oral glucose tolerance test (OGTT), as previously described [29], was conducted on mice at the $20^{\text {th }}$ week after the lycopene supplementation for 8 weeks. The glucose levels were measured at $0,30,60$, 90 , and $120 \mathrm{~min}$ after oral administration of glucose $(2 \mathrm{~g} /$ $\mathrm{kg}$ ) using a glucometer (Contour Plus, Bayer).

2.5. Serum Biomarkers Analysis. Serum levels of triglycerides (TG), total cholesterol (TC), high-density lipoprotein (HDL), low-density lipoprotein (LDL), T-AOC, SOD, and MDA were determined using the commercial kits according to the manufacturer's protocols.

2.6. Hematoxylin/Eosin (H\&E) and Tartrate-Resistant Acid Phosphatase (TRAP) Stainings. The paraffin-embedded sections ( $\sim 5 \mu \mathrm{m}$ thickness) from left femurs of the mice were processed as previously described [30]. The H\&E staining was performed according to the routine procedure.

TRAP staining was conducted as follows: the deparaffinized and rehydrated sections were incubated with Naphthol AS-BI phosphate solution (basic solution) for $45 \mathrm{~min}$ at $37^{\circ} \mathrm{C}$. After that, the slides were incubated with the basic solution with brown dyes (sodium nitrite and parafuchsin) for $6 \mathrm{~min}$ at room temperature followed by hematoxylin staining. 
TABle 1: Study design.

\begin{tabular}{llcc}
\hline Group & $\mathrm{n}$ & Diet & Intervention \\
\hline Normal control (NC) & 9 & Regular chow & Equal volume of sunflower oil \\
HFD-C & 9 & High-fat diet & Equal volume of sunflower oil \\
LYC & 9 & High-fat diet & Lycopene $(15 \mathrm{mg} / \mathrm{kg}$, dissolved in sunflower oil) \\
MET & 9 & High-fat diet & Metformin $(500 \mathrm{mg} / \mathrm{kg}$, dissolved in sunflower oil) \\
\hline
\end{tabular}

After staining, the histopathological alterations of trabecular bone were photographed and determined using an Olympus BX53 fluorescence microscope (Tokyo, Japan). The numbers of osteoclasts (OCs) were counted from the images.

2.7. Microcomputed Tomography ( $\mu$-CT) Analysis of Tibias. $\mu$-CT scanning was performed on the left tibia as previously described [31]. The alterations of the proximal tibial metaphysis and diaphysis were examined. The following parameters in the volume of interest were analyzed by the Analyzer Software (V12.0) as shown in Table $2[32,33]$.

2.8. Bone Biomechanical Strength and Material Profile Assays. After $\mu$-CT scanning, the right tibias were used for a three-point bending examination by an electronic universal testing machine (Brookfield CT3, America) as previously described [30].

After that, the tibia was triturated under liquid nitrogen in a ceramic mortar. The spectrum was obtained by a Fourier transform infrared spectroscopy (FTIR; Bruker Vertex 70, Germany). Scanning was conducted in transmission mode $\left(4,000-400 \mathrm{~cm}^{-1}\right.$ range, $4 \mathrm{~cm}^{-1}$ resolution, and 64 scans) [34]. The following parameters were determined as follows: (1) collagen maturity, the relative intensity ratios of the $1,660^{-1}$ to $1,690 \mathrm{~cm}^{-1}$ peaks [35]; (2) mineral maturity/crystallinity, the intensity ratio of $1030 \mathrm{~cm}^{-1}$ to $1020 \mathrm{~cm}^{-1}$ [36]; (3) the relative ratio of mineralization to the collagen matrix (mineral/matrix) [37]; and (4) the relative ratio of carbonate to phosphate, the area ratio v1, v3 band to $22 \mathrm{CO}_{3}[38,39]$.

2.9. Immunohistochemical (IHC) Analysis. IHC staining was conducted as previously described [31]. Briefly, the slides were incubated with the appropriate primary antibody [AGEs (1:50), RAGE $(1: 100)$, p-NF-кBp65 (1:100), NFKBp65 $(1: 100)$, and catK $(1: 100)]$ overnight at $4^{\circ} \mathrm{C}$ followed by the corresponding secondary antibody. Subsequently, slides were examined and photographed using an Olympus BX53 microscopy. The intensity of DAB staining was analyzed using an Image Pro Plus6.0 software and expressed as IOD value.

2.10. Western Blot Assay. The protein samples were prepared and transferred to the membranes as previously described [16]. The primary antibodies were as follows: AGEs (1:500), RAGE $(1: 1,000)$, p-NF-кBp65 (1:500), NF-кBp65 $(1: 500)$, GAPDH $(1: 20,000)$, and $\beta$-actin $(1: 5,000)$. The images captured with Azure Bioimaging systems were quantified using the Image J software and normalized with the corresponding $\beta$-actin or GAPDH.
2.11. Statistical Analysis. Data statistical analyses and graphical representations were conducted using GraphPad Prism (version 6.0, GraphPad Software, Inc., USA). All the data were presented as mean $\pm \mathrm{SD}$. When the data met homogeneity of variance and normality, a one-way analysis of variance (ANOVA) was employed. A Dunnett's T3 test or nonparametric test was applied, respectively, when the data met a normal distribution, but the homogeneity of variances was not achieved, or did not meet a normal distribution. Pearson's correlation analysis was used to examine the correlations between redox indicators and bone quality markers. Statistical significance was determined at $p<0.05$.

\section{Results}

3.1. Lycopene Improves Glucose and Lipid Metabolism in Obese Mice. As illustrated in Table 3, HFD exposure significantly increased body weight and fasting blood glucose levels in ICR mice. In detail, the body weight of the mice increased by $\sim 17.2 \%, \sim 16.7 \%$, and $\sim 18.5 \%$, respectively, in the groups of HFD-C, HFD + MET, and HFD + LYC relative to that of the NC group after 12 weeks' HFD exposure. Moreover, at the $22^{\text {nd }}$ week, the body weight of the mice in the HFD-C group increased by $\sim 33 \%$ compared to that in the NC group. Interestingly, metformin and lycopene intervention significantly inhibited body weight gain by $\sim 5.5 \%$ and $\sim 4.7 \%$ in obese mice $(p<0.05)$.

As for blood lipids, mice in the HFD-C group displayed obvious elevations in TG $(\sim 33 \%)$, TC $(\sim 77 \%)$, and LDL $(\sim 4-$ fold) and a significant decrease in HDL $(\sim 42 \%)$ relative to those in the NC group $(p<0.05)$. Notably, mice in the MET and LYC groups exhibited a relative lower level of TG ( 23\%), TC ( 20\%), and LDL ( 65\%) and a higher level of HDL $(\sim 42 \%)$ than those in the HFD-C group.

In addition, at the $20^{\text {th }}$ week, the blood glucose levels of the mice in the HFD-C group at $0,30,60,90$, and $120 \mathrm{~min}$ were higher than those in the NC group by OGTT assay (Table 4). Moreover, at the $22^{\text {nd }}$ week, the fasting blood glucose (FBG) levels of the mice in the HFD-C group were increased by $\sim 53 \%$ relative to those in the NC group (Table 3). These alterations were reversed by LYC and MET intervention as evidenced by $~ 20 \%$ and 25\% decrease in the FBG levels, respectively, in comparison with those in the HFD-C group $(p<0.05)$. Together, these results suggest that lycopene could stabilize blood glucose and lipid metabolism in obese mice.

3.2. Lycopene Preserves Bone Microarchitecture, Strength, and Material Profiles in Obese Mice. To examine whether lycopene could protect bone microarchitecture from HFD 
TABLE 2: The trabecular bone structural parameters analyzed by $\mu$-CT.

\begin{tabular}{lcc}
\hline Parameter & Full name & Meaning \\
\hline BMD & Bone mineral density & Total bone mineral content divided by the total volume \\
BS/TV & Bone volume fraction & Tone surface density \\
Tb.N & Trabecular number & The ratio of the segmented bone volume to the total volume \\
Tb.Sp & Trabecular separation & The bone surface area per total volume \\
Tb.Th & Trabecular thickness & The number of trabecular in the region of interest \\
Conn.D & Connectivity density & The distance between trabecular bones measured by 3D construction \\
SMI & Structure model index & The thickness of trabecular bone \\
Tt.Ar & Total cross-sectional area & The proportion between plate-like structure and rod-like structure in trabecular bone \\
Ct.Ar & Cortical bone area & Total area of cortical bone \\
Ct.Th & Cortical bone thickness & Cross-sectional area of the cortical bone \\
Ma.Ar & Bone marrow area & The thickness of cortical bone
\end{tabular}

TABLE 3: Lycopene reduces body weight gain and improves glucose and lipid metabolism in HFD-induced obese mice $(x \pm$ SD).

\begin{tabular}{|c|c|c|c|c|}
\hline Parameters & Normal & HFD-C & HFD + MET & HFD + LYC \\
\hline BW (0 week, g) & $20.00 \pm 2.00$ & $20.00 \pm 2.00$ & $20.00 \pm 2.00$ & $20.00 \pm 2.00$ \\
\hline BW $\left(12^{\text {th }}\right.$ week, g) & $46.37 \pm 1.79$ & $54.37 \pm 7.38^{\#}$ & $54.15 \pm 5.94^{\#}$ & $54.93 \pm 4.65^{\#}$ \\
\hline BW $\left(22^{\text {nd }}\right.$ week, g) & $44.02 \pm 2.78$ & $66.18 \pm 7.28^{\#}$ & $49.29 \pm 6.41^{*}$ & $52.33 \pm 7.71^{*}$ \\
\hline $\mathrm{TG}(\mu \mathrm{M})$ & $1.02 \pm 0.17$ & $1.35 \pm 0.08^{\#}$ & $1.04 \pm 0.08^{*}$ & $1.13 \pm 0.05^{*}$ \\
\hline $\mathrm{TC}(\mu \mathrm{M})$ & $4.30 \pm 0.36$ & $7.6 \pm 0.97^{\#}$ & $6.10 \pm 0.86^{*}$ & $6.14 \pm 0.84^{*}$ \\
\hline $\mathrm{LDL}(\mu \mathrm{M})$ & $0.49 \pm 0.15$ & $2.56 \pm 0.33^{\#}$ & $0.90 \pm 0.32^{*}$ & $0.91 \pm 0.30^{*}$ \\
\hline HDL $(\mu \mathrm{M})$ & $5.44 \pm 0.97$ & $3.83 \pm 0.66^{\#}$ & $5.45 \pm 0.97^{*}$ & $6.36 \pm 0.90^{*}$ \\
\hline FBG $\left(12^{\text {th }}\right.$ week, mM/L $)$ & $5.35 \pm 0.98$ & $8.13 \pm 0.86$ & $8.68 \pm 2.56$ & $8.05 \pm 0.84$ \\
\hline FBG $\left(22^{\text {nd }}\right.$ week, $\left.\mathrm{mM} / \mathrm{L}\right)$ & $5.92 \pm 0.80$ & $9.06 \pm 1.01^{\#}$ & $7.23 \pm 0.39^{*}$ & $6.76 \pm 0.90^{*}$ \\
\hline
\end{tabular}

Notes: BW, body weight; FBG, fasting blood glucose; TG, triglycerides; TC, total cholesterol; HDL, high-density lipoprotein; LDL, low-density lipoprotein; HFD-C, high-fat diet-fed control; MET, metformin treatment; LYC, lycopene treatment; data are presented as mean \pm SD. ${ }^{*}$ vs the NC group. ${ }^{*}$ vs the HFD-C group. $p<0.05$ was considered statistical difference.

TABLE 4: The mice blood glucose levels at $20^{\text {th }}$ week $(0,30,60,90$, and $120 \mathrm{~min})(x \pm \mathrm{SD})$.

\begin{tabular}{lcccc}
\hline Time $(\mathrm{min})$ & Normal & HFD-C & HFD + MET & HFD + LYC \\
\hline 0 & $3.79 \pm 1.49$ & $6.28 \pm 0.84^{\#}$ & $4.78 \pm 1.11^{*}$ & $3.04 \pm 0.30^{*}$ \\
30 & $10.08 \pm 1.06$ & $15.1 \pm 2.99^{\#}$ & $11.12 \pm 4.31^{*}$ & $11.72 \pm 1.20^{*}$ \\
60 & $7.17 \pm 1.12$ & $11.57 \pm 1.11^{\#}$ & $9.26 \pm 0.35^{*}$ & $8.44 \pm 0.68^{*}$ \\
90 & $5.77 \pm 1.17$ & $8.55 \pm 0.85^{\#}$ & $7.01 \pm 1.87$ & $5.38 \pm 1.24^{*}$ \\
120 & $4.18 \pm 0.87$ & $7.66 \pm 1.47^{\#}$ & $6.53 \pm 0.94$ & $5.08 \pm 0.94^{*}$ \\
\hline
\end{tabular}

Notes: HFD-C, high-fat diet-fed control; MET, metformin treatment; LYC, lycopene treatment; data are presented as mean \pm SD. ${ }^{*}$ vs the NC group. ${ }^{*}$ vs the HFD-C group. $p<0.05$ was considered statistical difference.

exposure, a $\mu$-CT assay was performed. As shown in Figures $1(\mathrm{a})-1(\mathrm{n})$, the $\mu$-CT images of the proximal tibial metaphysis and its analyses demonstrated that the mice in the HFD-C group exhibited a decline in BV/TV, BS/TV, Tb.N, Tb.Th, and Conn.D, as well as an increase in BMD, Tb.Sp, and SMI when compared to those in the NC group (Figures 1(b)-1(i); $p<0.05)$. As for the tibial diaphysis, mice in the HFD-C group presented a decrease in BMD, Tt.Ar, and Ct.Th and an increase in Ct.Ar and Ma.Ar when compared to those in the NC group (Figures $1(\mathrm{j})-1(\mathrm{n}) ; p<$ 0.05). Interestingly, the administration of metformin or lycopene to obese mice reversed the aforementioned alterations of bone microstructure $(p<0.05)$ except for Ct.Ar in the tibial diaphysis. 
NC
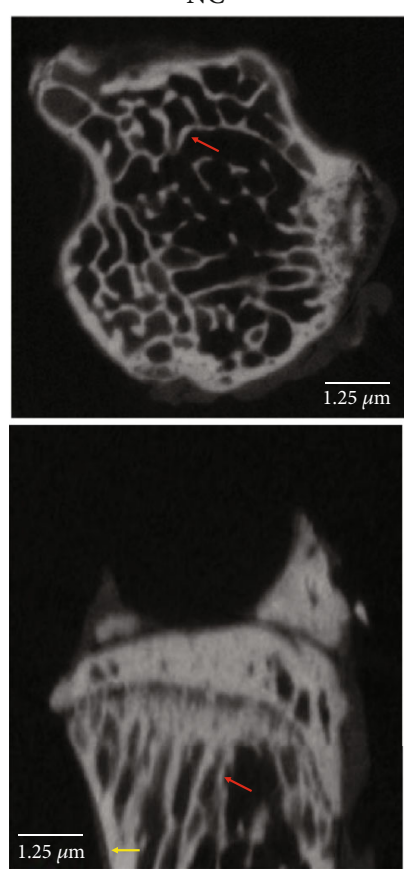

HFD-C
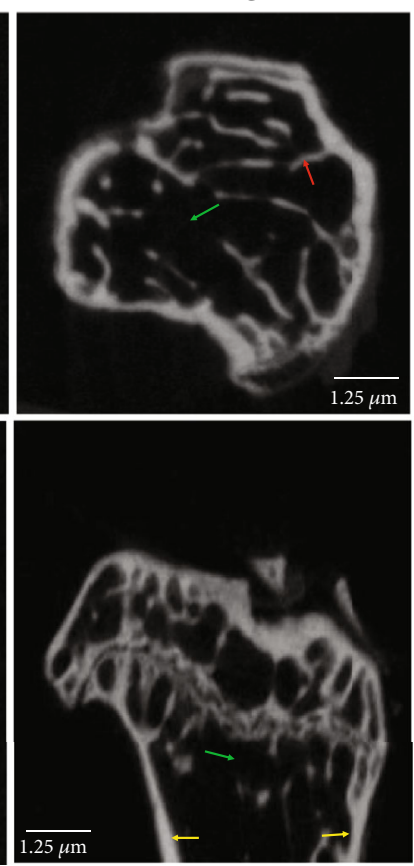

MET
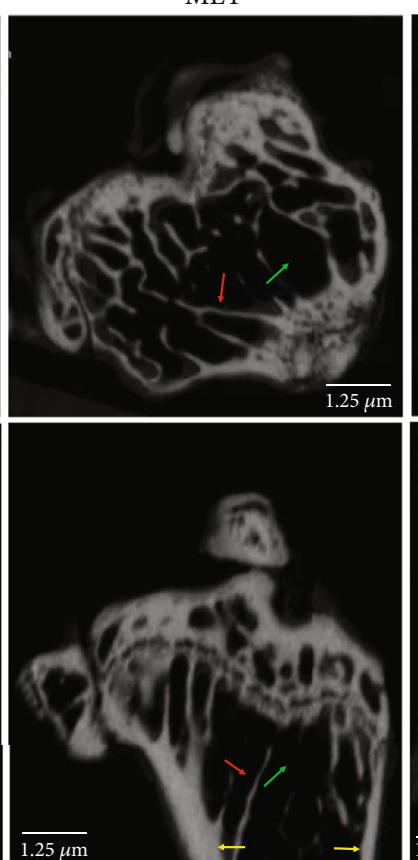

(a)
LYC
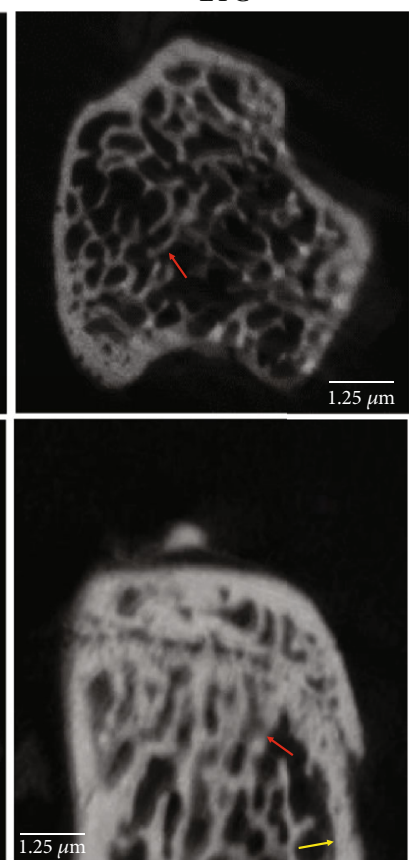

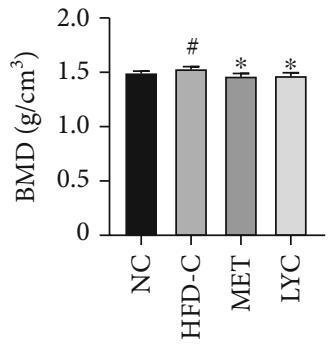

(b)

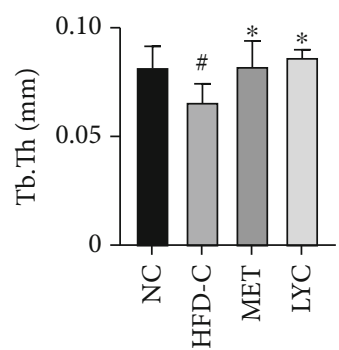

(e)

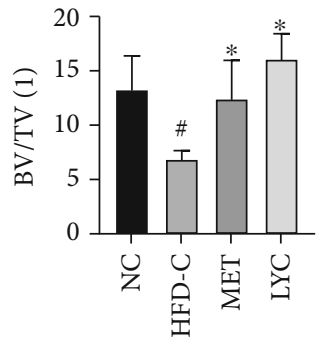

(c)

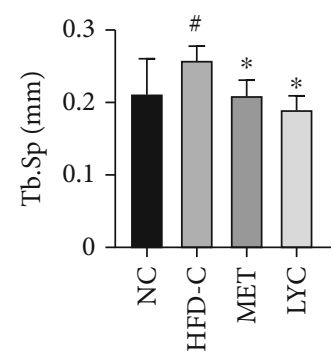

(f)

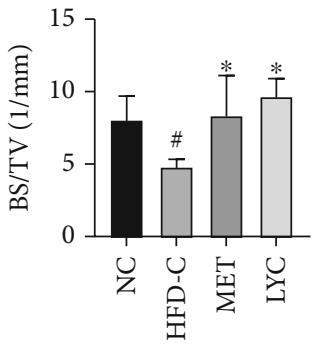

(d)

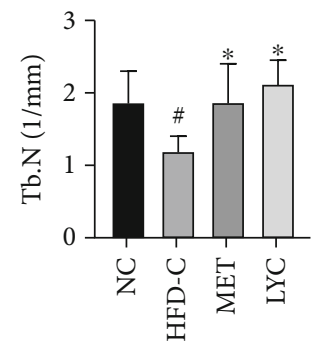

(g)

Figure 1: Continued. 


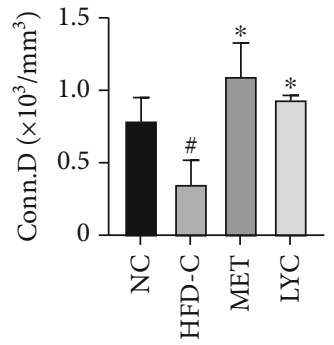

(h)

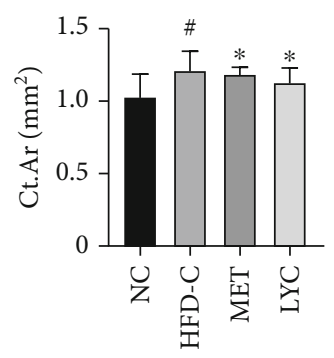

(l)

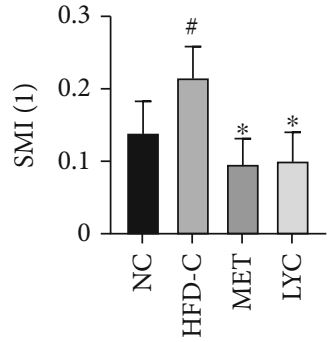

(i)

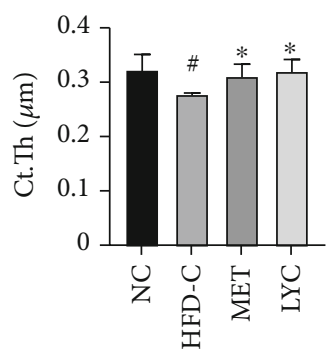

(m)

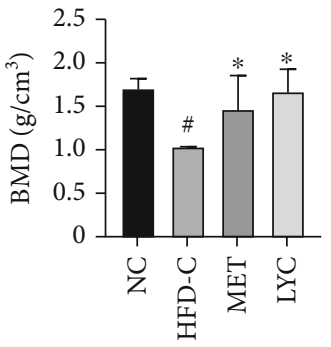

(j)

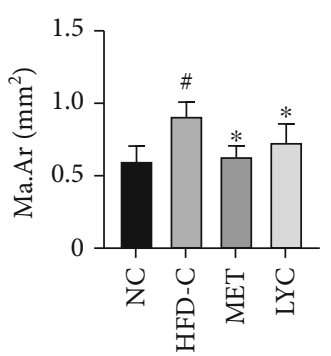

(n)

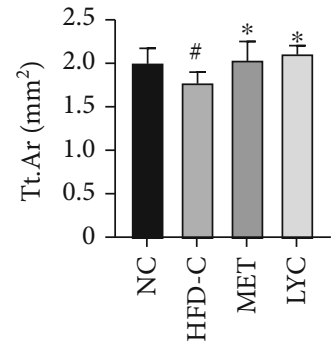

(k)

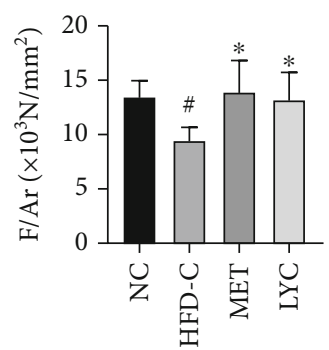

(o)

FIGURE 1: Lycopene preserves bone microarchitecture and strength in HFD-induced obese mice. The representative microimages of the transverse and longitudinal sections in the proximal tibia were obtained from micro $(\mu)$-CT (the red arrow indicates trabecular bone; the green arrow indicates bone marrow cavity. The yellow arrow indicates cortical bone) (a). $\mu$-CT-derived quantification data of tibial metaphysis including BMD (b), BV/TV (c), BS/TV (1/mm) (d), Tb.Th (mm) (e), Tb.Sp (mm) (f), Tb.N (1/mm) (g), Conn.D (1/mm $\left.{ }^{3}\right)$ (h), and SMI (i). The quantification data of tibial diaphysis including BMD (j), Tt.Ar $\left(\mathrm{mm}^{2}\right)(\mathrm{k}), \mathrm{Ct} . \mathrm{Ar}\left(\mathrm{mm}^{2}\right)(\mathrm{l}), \mathrm{Ct} . \mathrm{Th}(\mathrm{um})(\mathrm{m})$, and Ma.Ar $\left(\mathrm{mm}^{2}\right)(\mathrm{n})$. The ultimate load in the tibia, F/Ar $\left(\mathrm{N} / \mathrm{mm}^{2}\right)$ (o). Data are presented as mean \pm SD. LYC denotes lycopene treatment; MET denotes metformin treatment. ${ }^{*} v$ s the NC group. ${ }^{*} v s$ the HFD-C group. $p<0.05$ was considered statistically significant.

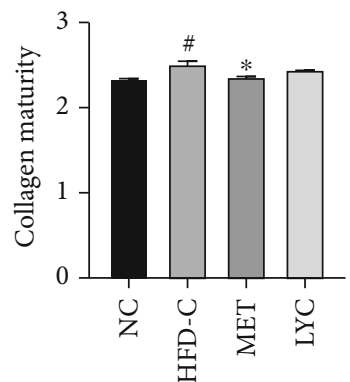

(a)

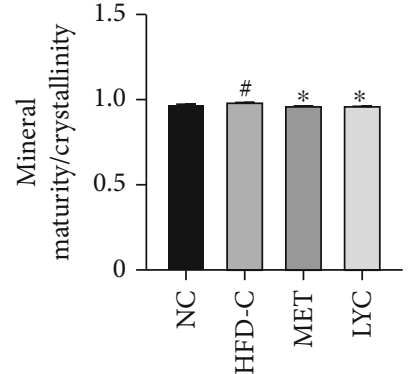

(b)

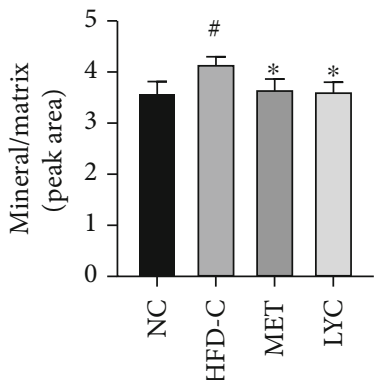

(c)

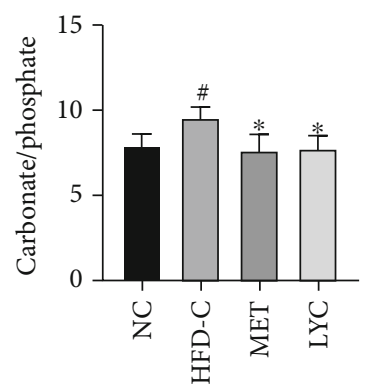

(d)

FIGURE 2: Lycopene preserves bone material profiles in HFD-induced obese mice. Collagen maturity (a), mineral maturity/crystallinity (b), collagen matrix (mineral/matrix, (c)), and carbonate to phosphate (d) were determined by Fourier transform infrared spectroscopy (FTIR). LYC denotes lycopene treatment; MET denotes metformin treatment. Data are presented as mean \pm SD. ${ }^{*} v s$ the NC group. * $v s$ the HFD-C group. $p<0.05$ was considered statistically significant.

To further evaluate the action of lycopene on bone biomechanical properties, mice tibias were subjected to the three-point bending assay. As shown in Figure 1(o), the ultimate load of the mice in the HFD-C group was notably lower than that in the $\mathrm{NC}$ one $(p<0.05)$. As expected, the administration of lycopene or metformin to HFD-exposed obese mice inhibited a decline in the ultimate load in the tibias $(p<0.05)$.

Next, we analyzed the bone material alterations, including collagen maturity, mineral maturity/crystallinity, collagen matrix (mineral/matrix), and carbonate/phosphate in the tibias by FTIR. As shown in Figures 2(a)-2(d), the collagen maturity, the relative ratios of mineral maturity to crystallinity, mineral to matrix, and carbonate to phosphate were notably raised in the HFD-C group relative to the NC group $(p<0.05)$. The treatment with lycopene prevented the alterations in the tibias of HFD-exposed mice $(p<0.05)$ except for collagen maturity. Notably, metformin treatment reversed all the alterations in the tibias of HFD-exposed ones. These results suggest that lycopene not only maintains bone strength and microstructure but also improves bone material profiles in obese mice.

\subsection{Lycopene Improves Femoral Histopathological} Alterations in Obese Mice. To observe bone morphological alterations, H\&E and TRAP stainings were performed. As illustrated in Figure 3(a), the trabecular bone in the distal femurs of the mice in the HFD-C group lost regular mesh structure and 


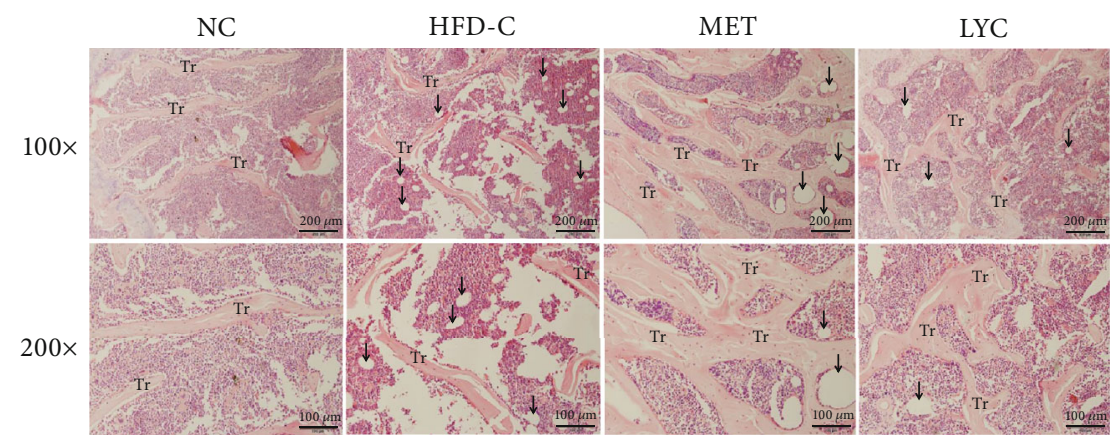

(a)

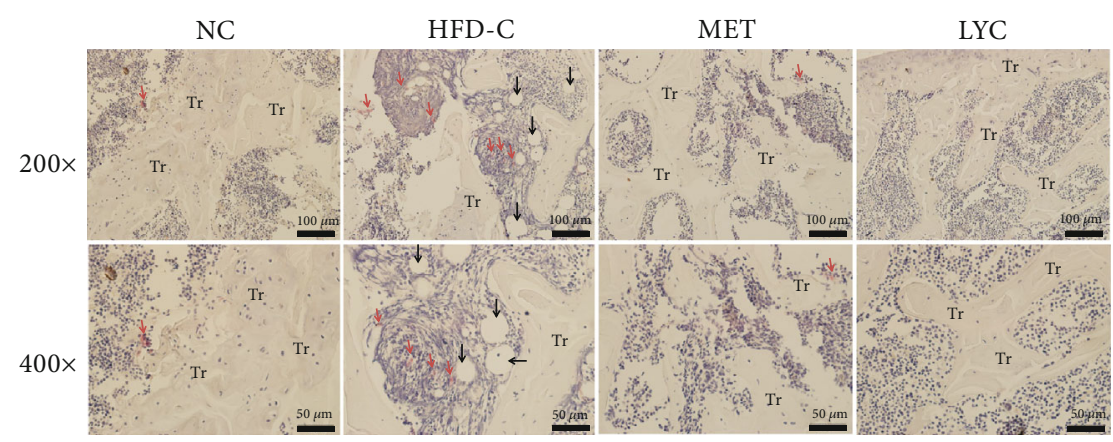

(b)

FIGURE 3: Lycopene improves bone histomorphological features in HFD-induced obese mice. Representative images of H\&E (a) and TRAP (b) staining in the femoral metaphysis of different groups of mice. The black arrow in panel (a) indicates lipid droplet. The red arrow in panel (b) indicates osteoclast. Tr denotes trabecular bone. LYC denotes lycopene treatment; MET denotes metformin treatment.

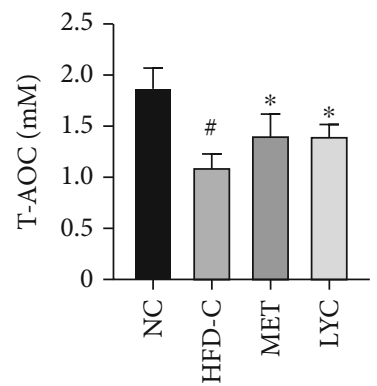

(a)

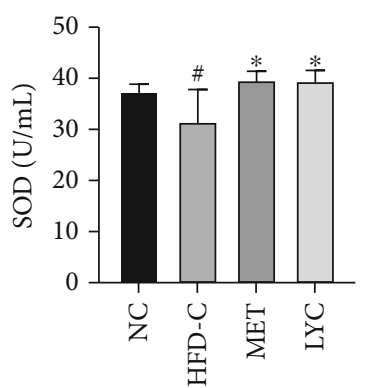

(b)

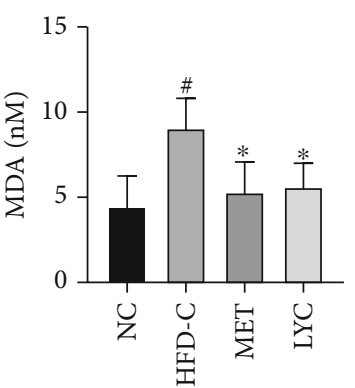

(c)

FIGURE 4: Lycopene ameliorates oxidative stress in HFD-induced obese mice. Serum levels of oxidative stress markers were determined by biochemical assays, including total antioxidant capacity (T-AOC, (a)), superoxide dismutase (SOD, (b)), and malondialdehyde (MDA, (c)). LYC denotes lycopene treatment; MET denotes metformin treatment. Data are presented as mean \pm SD. ${ }^{*} v s$ the $\mathrm{NC}$ group. ${ }^{*} v s$ the HFD-C group. $p<0.05$ was considered statistically significant.

became thinner and irregular as compared to the mice in the NC group. Furthermore, the lipid droplets (indicated by the black arrow) were much more obvious in the proximal femurs of HFD-C group. As expected, lycopene or metformin treatment improved femoral histopathological alterations.

As shown in Figure 3(b), the number of OCs was identified by TRAP staining (indicated by the red arrow). The mice in the HFD-C group exhibited an increased number of OCs in the femurs $(p<0.05)$. Notably, the trend was prevented by lycopene or metformin intervention $(p<0.05)$.

3.4. Lycopene Alleviates Oxidative Stress in Obese Mice. As shown in Figures 4(a)-4(c), serum levels of T-AOC and SOD were diminished, and serum levels of MDA were ele- vated in the mice of the HFD-C group, respectively, as compared to those in the NC group $(p<0.05)$. Interestingly, lycopene or metformin supplementation reversed these HFD-induced alterations in obese mice $(p<0.05)$. These results indicate that lycopene may alleviate oxidative stress in obese mice.

In addition, the correlation between blood markers and bone quality parameters was analyzed using Pearson correlation analysis. As shown in Table 5, the results showed that bone quality parameters were negative correlated with serum levels of TG, TC, LDL, and MDA and positively correlated with serum levels of $\mathrm{HDL}$, SOD, and T-AOC. In detail, there were significant positive correlations between TG, TC, T-AOC, and BMD of proximal tibial metaphysis, 
TABLE 5: The correlation between serum markers and bone quality.

\begin{tabular}{|c|c|c|c|c|c|c|c|}
\hline Parameters & TG & TC & LDL & HDL & MDA & SOD & T-AOC \\
\hline $\mathrm{BMD}(r)$ & 0.860 & 0.824 & 0.217 & -0.344 & -0.045 & 0.552 & 0.888 \\
\hline$p$ value & $0.027^{\#}$ & $0.043^{\#}$ & 0.680 & 0.505 & 0.932 & 0.256 & $0.018^{\#}$ \\
\hline $\mathrm{BV} / \mathrm{TV}(r)$ & -0.273 & -0.146 & -0.821 & 0.357 & -0.775 & 0.612 & 0.271 \\
\hline$p$ value & 0.553 & 0.708 & 0.067 & 0.488 & $-0.041^{\#}$ & 0.080 & 0.480 \\
\hline $\mathrm{BS} / \mathrm{TV}(r)$ & -0.285 & -0.105 & -0.508 & 0.119 & -0.733 & 0.578 & 0.184 \\
\hline$p$ value & 0.553 & 0.788 & 0.163 & 0.821 & 0.061 & 0.103 & 0.637 \\
\hline Tb.Th $(r)$ & -0.027 & -0.138 & -0.556 & 0.737 & -0.662 & 0.210 & 0.042 \\
\hline$p$ value & 0.953 & 0.723 & 0.120 & 0.095 & 0.095 & 0.587 & 0.587 \\
\hline Tb.Sp $(r)$ & 0.468 & 0.174 & 0.232 & -0.215 & 0.385 & -0.912 & -0.215 \\
\hline$p$ value & 0.289 & 0.289 & 0.548 & 0.683 & 0.394 & $0.001^{\#}$ & 0.579 \\
\hline Tb.N $(r)$ & -0.155 & -0.06 & -0.565 & 0.206 & -0.837 & 0.531 & 0.018 \\
\hline$p$ value & 0.738 & 0.872 & 0.113 & 0.113 & $0.019^{\#}$ & $0.019^{\#}$ & 0.964 \\
\hline Conn.D $(r)$ & -0.309 & -0.106 & -0.206 & 0.369 & 0.354 & -0.295 & -0.111 \\
\hline$p$ value & 0.499 & 0.784 & 0.594 & 0.471 & 0.471 & 0.471 & 0.776 \\
\hline SMI $(r)$ & 0.527 & 0.398 & 0.096 & -0.576 & -0.252 & 0.815 & 0.109 \\
\hline$p$ value & 0.223 & 0.288 & 0.288 & 0.231 & 0.586 & $0.007^{\#}$ & $0.007^{\#}$ \\
\hline $\mathrm{BMD}(\mathrm{r})$ & -0.612 & -0.607 & -0.278 & 0.213 & -0.804 & 0.679 & 0.560 \\
\hline$p$ value & 0.144 & 0.082 & 0.468 & 0.468 & $0.012^{\#}$ & $0.012^{\#}$ & 0.117 \\
\hline Tt.Ar $(r)$ & -0.749 & -0.183 & -0.384 & 0.133 & -0.498 & 0.027 & 0.216 \\
\hline$p$ value & 0.052 & 0.637 & 0.637 & 0.801 & 0.801 & 0.801 & 0.577 \\
\hline Ct.Ar $(r)$ & 0.534 & 0.074 & 0.209 & -0.032 & 0.650 & -0.013 & -0.070 \\
\hline$p$ value & 0.216 & 0.849 & 0.589 & 0.952 & 0.114 & 0.973 & 0.973 \\
\hline Ct.Th $(r)$ & -0.095 & -0.352 & -0.108 & 0.08 & -0.682 & 0.052 & 0.401 \\
\hline$p$ value & 0.839 & 0.351 & 0.781 & 0.781 & 0.781 & 0.894 & 0.894 \\
\hline Ma.Ar $(r)$ & 0.847 & 0.372 & 0.452 & -0.212 & 0.209 & -0.058 & -0.419 \\
\hline$p$ value & $0.016^{\#}$ & 0.324 & 0.221 & 0.687 & 0.653 & 0.883 & 0.261 \\
\hline $\mathrm{F} / \mathrm{Ar}(r)$ & -0.332 & -0.251 & -0.033 & 0.306 & -0.014 & 0.012 & 0.184 \\
\hline$p$ value & 0.466 & 0.515 & 0.933 & 0.933 & 0.976 & 0.974 & 0.635 \\
\hline
\end{tabular}

Notes: TG, triglycerides; TC, total cholesterol; HDL, high-density lipoprotein; LDL, low-density lipoprotein; HFD-C, high-fat diet-fed control; MET, metformin; LYC, lycopene; T-AOC, total antioxidant capacity; SOD, superoxide dismutase; MDA, malondialdehyde; BMD, bone mineral density; BV/TV, bone volume fraction; BS/TV, bone surface density; Tb.N, trabecular number; Tb.Sp, trabecular separation; Tb.Th, trabecular thickness; Conn.D, connectivity density; SMI, structure Model Index; Tt.Ar, total cross-sectional area; Ct.Ar, cortical area; Ct.Th, cortical thickness; Ma.Ar, marrow area. \#, $p$ $<0.05$ was considered statistical difference.

between Tb.N, SMI, and BMD of tibial diaphysis and SOD, between Ma.Ar of tibial diaphysis and TG, and between SMI and T-AOC. In addition, there were significant negative correlations between BV/TV, Tb.N, BMD of tibial diaphysis, and MDA. These results suggested that blood glucose and lipid metabolism could affect bone quality.

3.5. Lycopene Inhibits the Expressions of AGEs, RAGE, NF$\kappa B$, and catK in the Femurs and Tibias of Obese Mice. Persistent hyperglycemia results in an over-accumulation of AGEs, which enhance ROS generation and contribute to NF- $\mathrm{\kappa B}$ activation, ultimately leading to bone resorption via RAGE [40, 41]. As shown in Figures 5(a)-5(f), the expressions of AGEs and RAGE in the femurs and tibias were notably increased in the mice of the HFD-C group when compared to those in the NC group $(p<0.05)$. And lycopene treatment significantly reduced the expres- sions of AGEs and RAGE in the hindlimbs of obese mice compared to those of the vehicle-treated controls $(p<0.05$ ) .

Moreover, as shown in Figures 6(a)-6(l), the expressions of NF-кBp65, p-NF-кBp65, and catK, as well as the nuclei ratios of $\mathrm{p}-\mathrm{NF}-\mathrm{\kappa Bp} 65$ to $\mathrm{NF}-\mathrm{\kappa Bp} 65$ were significantly increased in the femurs and tibias of the HFD-C group of mice compared with those in the NC group $(p<0.05)$. Intriguingly, lycopene treatment markedly reduced the expressions of NF-кBр65, р-NF-кBр65, catK, and the nuclei ratio of $\mathrm{p}-\mathrm{NF}-\kappa \mathrm{Bp} 65$ to NF-кBp65 in the tibias and femurs of obese mice $(p<0.05)$. However, there were no differences in the relative ratios of $\mathrm{p}-\mathrm{NF}-\mathrm{\kappa} B \mathrm{p} 65$ to NF-кBp65 in the cytoplasm of the bones amongst the three groups. These results suggest that lycopene may regulate the AGE/RAGE/ROS/NF- $\mathrm{BB}$ signaling pathway in HFD-induced obese mice. 


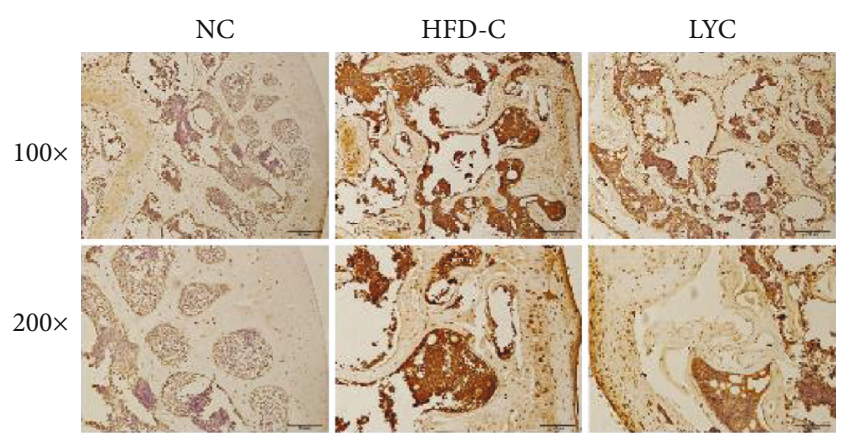

(a)

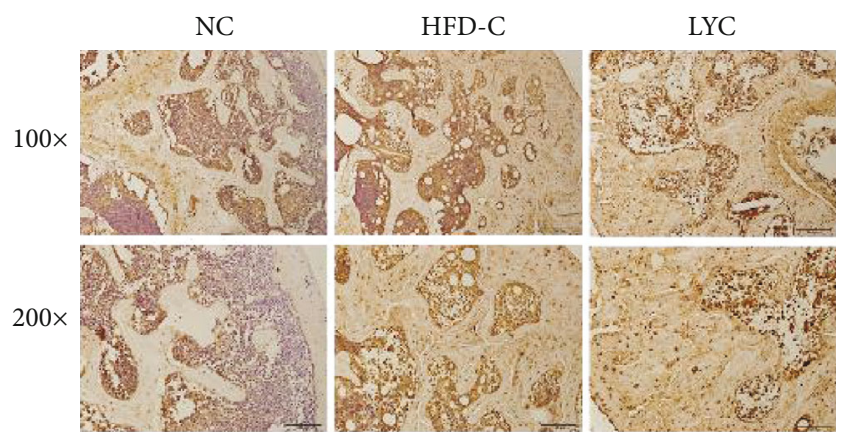

(d)

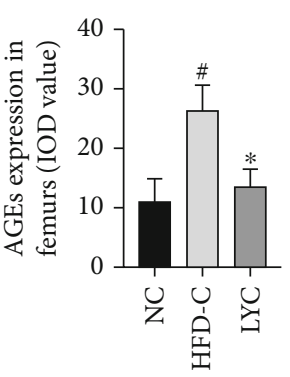

(b)

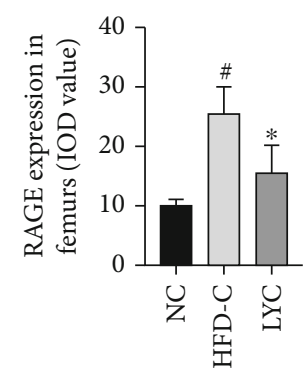

(e)

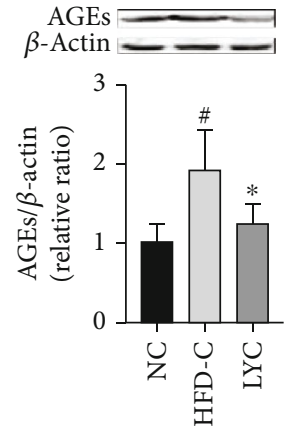

(c)

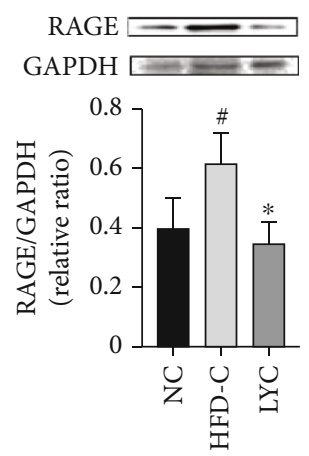

(f)

FIGURE 5: Lycopene inhibits the expressions of AGEs and RAGE in the femurs and tibias of HFD-induced obese mice. The expressions of AGEs and RAGE were analyzed by immunohistochemical staining ( $a, b, d$, and e) and western blot (c and f). LYC denotes lycopene treatment; MET denotes metformin treatment. Data are presented as mean \pm SD. ${ }^{*} v s$ the NC group. ${ }^{*} v s$ the HFD-C group. $p<0.05$ was considered statistically significant.

\section{Discussion}

In the present study, we demonstrated that lycopene reduces body weight gain and improves blood glucose and lipid metabolism in obese mice. Secondly, lycopene treatment reverses obesity-induced alterations in the bone microarchitecture, biomechanical strength, and material profiles. Thirdly, lycopene intervention increases serum levels of TAOC and SOD and decreases serum levels of MDA in obese mice. In addition, we provided the evidence of lycopene improving bone metabolism by inhibiting obesity-induced upregulation of AGEs, RAGE, p-NF-кBp65, NF-кBp65, рNF-кBр65/NF-кBр65, and catK in the femurs and tibias of obese mice.

Lycopene was shown to reduce body weight gain and improve blood glucose and lipid metabolism in obese mice, which is in line with our previous study [29]. It is known that the sustained dyslipidemia and hyperglycemia induce an increase in adipogenesis and a decrease in osteogenesis [42-44], which lead to reduced bone formation. Moreover, ROS overproduction and dyslipidemia attenuate bone material profiles and bone strength [45-47], leading to a diminished bone quality. Indeed, we found that obese mice exhibit a decreased bone biomechanical strength and a disorganized bone microstructure. Interestingly, lycopene was reported to exert bone protective effect through its antioxidant properties $[24,48]$. In addition, as shown in the present study, lycopene treatment alleviates oxidative stress by increasing serum levels of T-AOC and SOD and decreasing serum levels of MDA in obese mice. The administration of lycopene for 10 weeks to obese mice results in an improvement in bone material profiles. Also, lycopene intervention increases the capacity of ultimate load in the tibias of obese mice. Together, these results suggested that lycopene has the ability of improving bone quality through the regulation of oxidative stress in obese mice.

The sustained high levels of ROS may be associated with heightened AGE expressions upon hyperglycemia stimulation [49], which further induces NF-кB activation and subsequent bone resorption $[50,51]$. We demonstrated that lycopene treatment decreases the expressions of AGEs and RAGE as well as inhibits NF-KBp65 activation in the femurs and tibias of obese mice. These results were in line with the findings that lycopene is able to decrease the levels of plasma AGEs and renal RAGE [52] and suppress NF- $\mathrm{KB}$ activation in hyperglycemia rats and HK-2 cells $[49,52,53]$. In addition, lycopene was reported to inhibit ROS-induced NF- $\mathrm{BB}$ activation in pancreatic cancer cells [54]. Moreover, dietary lycopene could decrease bone resorption in postmenopausal women $[26,55]$. In the present study, we demonstrated that lycopene decreases catK expression and inhibits bone resorption in obese mice. Together, these findings suggested that lycopene may improve bone quality through the regulation of the AGE/RAGE/ROS/NF- $\mathrm{KB}$ signaling pathway in HFD-induced obese mice.

The dyslipidemia in HFD-induced obese mice was normalized by lycopene intervention in the current study, which was associated with a reduction in AGE generation and NF- 


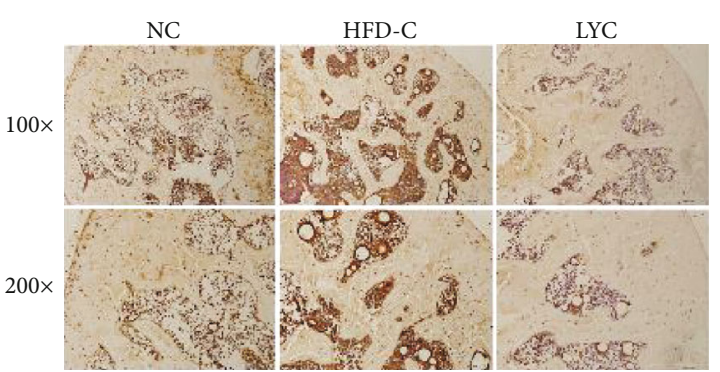

(a)

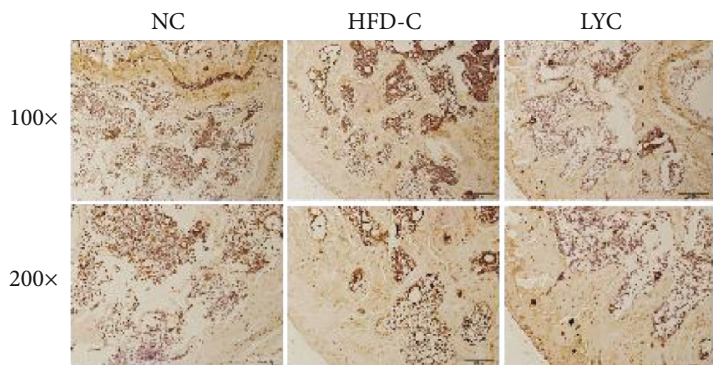

(e)

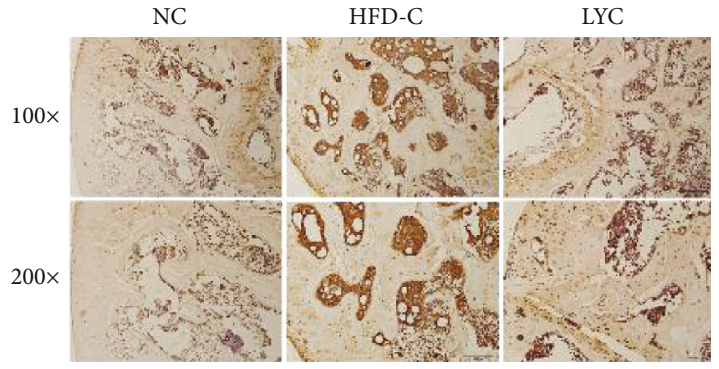

(i)

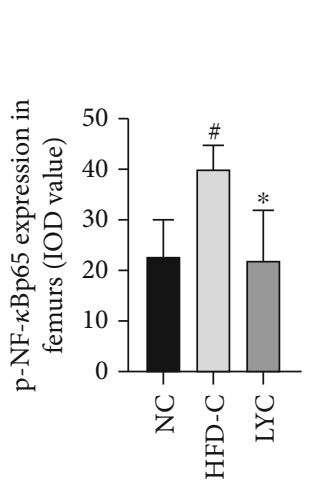

(b)

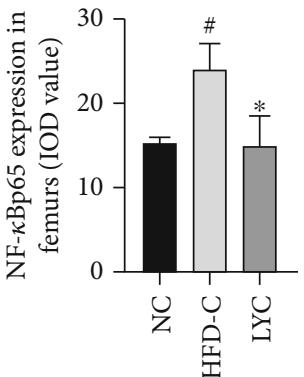

(f)

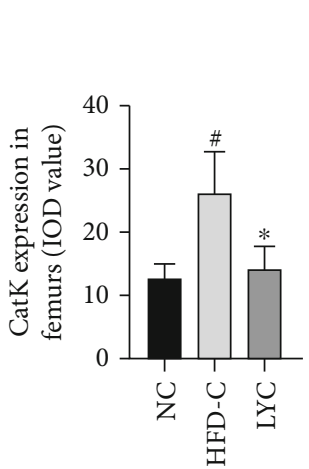

(j)

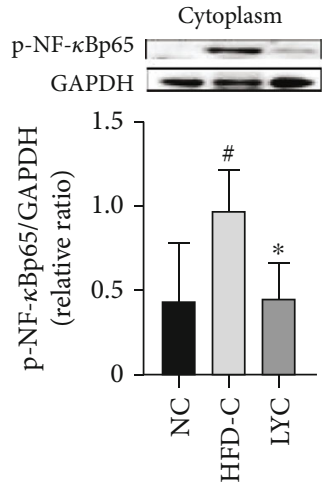

(c)

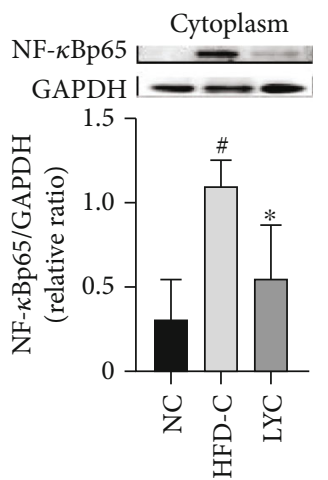

(g)

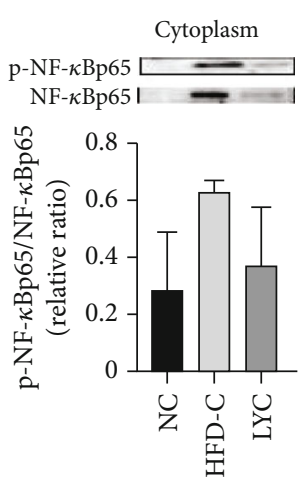

(k)

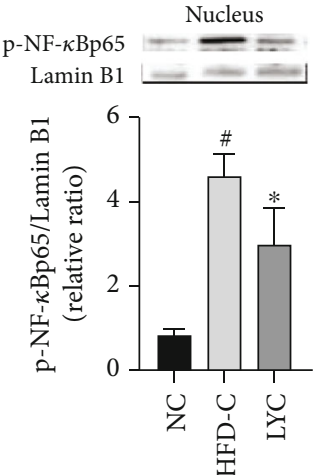

(d)

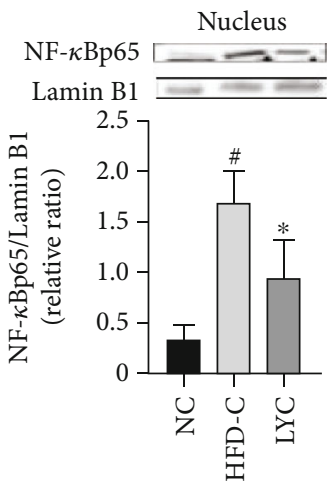

(h)

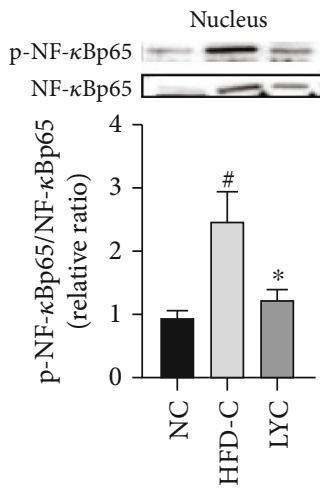

(l)

Figure 6: Lycopene suppresses the expressions of p-NF-кBр65, NF-кBр65, р-NF-кBр65/NF-кBр65, and catK in the femurs and tibias of

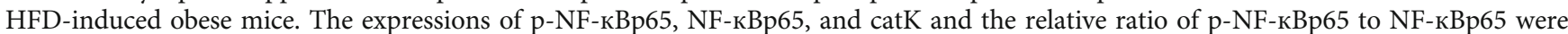
determined by immunohistochemical staining ( $a, b, e, f, e$, and j) and western blot (c, d, g, h, k, and l). LYC denotes lycopene treatment; MET denotes metformin treatment. Data are presented as mean \pm SD. ${ }^{*} v s$ the NC group. ${ }^{*} v s$ the HFD-C group. $p<0.05$ was considered statistical difference.

кВ activation. Notably, AGE overproduction contributes to dyslipidemia through reacting with plasma lipoproteins [21]. In addition, high cholesterol may activate NF-кB signaling through promotion of ROS production [56], which accelerates osteoclastic bone resorption and consequent osteoporosis [18]. Collectively, these findings suggest that lycopene may preserve bone remodeling through the regulation of lipid metabolism and inhibition of AGE production in obese mice.

In the present study, we demonstrated that HFDinduced hyperglycemia mice exhibit an increase in the BMD of the metaphysis and a decrease in the diaphysis of the tibias. This is in line with the observations from HoPham. et al. who reported that type 2 diabetes mellitus (T2DM) patients had higher trabecular BMD but lower cortical BMD [57]. It is generally accepted that patients with T2DM have normal or higher BMD in the femoral neck and spine $[58,59]$. However, an elevation of BMD in T2DM is associated with increased risk of bone fragility [60]. Indeed, disorganized bone microarchitectures, such as increased cortical porosity and decreased cortical bone density, account for an increased risk of fractures [57]. In addition, the compromised bone material profiles may attenuate bone quality [61]. Here, we demonstrated that the obesity- 
induced hyperglycemia mice exhibit abnormal tibia remodeling characterized by deteriorated microstructure and compromised material profiles, which were prevented by lycopene treatment. However, we only evaluated the alterations of the material profiles in the whole tibia powders. In the future, we will compare the differences of bone material profiles between epiphysis, metaphysis, and diaphysis in obese mice in response to lycopene treatment. Taken together, these findings suggested that lycopene consumption may improve bone quality through the regulation of bone microstructure and material profiles.

In our study, we confirmed that the disordered glucose and lipid metabolism in obese mice were restored by metformin treatment, which is in line with the investigations from the other groups $[62,63]$. In addition, the bone protective effect conferred by metformin was comparable to that of lycopene, including an improvement in the bone microstructure, biomechanical strength, and material profiles in obesity-induced hyperglycemia mice. These alterations are associated with the restorations of bone histomorphological architecture. Interestingly, Bornstein et al. reported that metformin was able to reverse the inhibition of osteoblastogenesis and to suspend bone marrow and inguinal adipose expansion in HFD-induced obese mice [64]. Notably, we demonstrated that metformin intervention inhibits an elevation of osteoclast activity and oxidative stress in obesityinduced hyperglycemia mice. In agreement with the current findings, metformin was reported to suppress osteoclast differentiation in collagen-induced arthritic mice [65] and inhibit ROS generation under osteoporotic conditions [66]. As mentioned above, an elevation of oxidative stress contributes to osteoclastic bone resorption rather than osteoblastic bone formation. Therefore, it is suggested that metformin may improve bone metabolism through the regulation of redox homeostasis. However, the underlying mechanism whereby metformin exerts antiosteoporotic effect still merits further investigation.

The current study still has limitations. We did not investigate the effect of metformin on the expression levels of the proteins involved in AGE/RAGE/NF- $\mathrm{KB}$ signaling pathway. However, metformin has been reported to inhibit the AGE formation [67]. In addition, a number of studies have reported that metformin could downregulate RAGE expression and reduce NF- $\kappa \mathrm{B}$ activation in various cells, including osteoblastic cells [68-70], MCF-7, MDA-MB-231 cells [71], and osteosarcoma stem cells [72]. Moreover, Bian et al. reported [73] that low concentration of metformin inhibited osteoclast differentiation, as well as downregulated catK expression, and its inhibitory effect was gradually enhanced with increasing concentrations of metformin. Therefore, the effects of metformin could be in dose-dependent manner and deserve to be addressed in the future study.

\section{Conclusion}

In summary, we demonstrated that lycopene improves bone quality in HFD-induced obese mice. The underlying mechanism might be associated with the regulation of the AGE/ RAGE/NF-кB signaling pathway. These results suggest that dietary supplementation of lycopene may offer a new therapeutic strategy for the management of obesity and its associated-osteoporosis, which needs to be further evaluated in clinical trials.

\section{Data Availability}

The data used to support the findings of this study are available from the corresponding authors upon request.

\section{Conflicts of Interest}

The authors declare no conflicts of interest regarding the publication of this paper.

\section{Authors' Contributions}

Bingke Xia, Ruyuan Zhu, Lili Wang, Xinxiang Wang, and Dongwei Zhang designed the study. Bingke Xia, Ruyuan Zhu, Hao Zhang, Beibei Chen, Yage Liu, Xuan Dai, Zimengwei Ye, Lili Wang, Xinxiang Wang, and Dongwei Zhang collected the data, designed and developed the database, carried out data analyses, and produced the initial draft of the manuscript. Dandan Zhao, Fangfang Mo, and Sihua Gao contributed to drafting the manuscript. Xiang-Dong Wang, Dieter Brömme, Lili Wang, Xinxiang Wang, and Dongwei Zhang revised and reviewed the manuscript. All authors have read and approved the final version of the manuscript. Bingke Xia and Ruyuan Zhu equally contributed to this study. Lili Wang, Xinxiang Wang, and Dongwei Zhang are co-corresponding authors.

\section{Acknowledgments}

This work was supported by the Grants from the National Natural Science Foundation of China (NSFC, Grants Nos. 81874373 and 82074235), the Fundamental Research Funds for the Central Universities (Grant No. 2021-JYB-XJSJJ007), and the United States Department of Agriculture (USDA/ARS, No.8050-51000-096-02S). The funding sources have no roles in study design, data analysis and interpretation, and paper submission.

\section{References}

[1] S. Mohsin, M. M. Baniyas, R. S. AlDarmaki, K. Tekes, H. Kalász, and E. A. Adeghate, "An update on therapies for the treatment of diabetes-induced osteoporosis," Expert Opinion on Biological Therapy, vol. 19, no. 9, pp. 937-948, 2019.

[2] Y. C. Chooi, C. Ding, and F. Magkos, "The epidemiology of obesity," Metabolism, vol. 92, pp. 6-10, 2019.

[3] K. Gkastaris, D. G. Goulis, M. Potoupnis, A. D. Anastasilakis, and G. Kapetanos, "Obesity, osteoporosis and bone metabolism," Journal of Musculoskeletal \& Neuronal Interactions, vol. 20, pp. 372-381, 2020.

[4] M. P. Czech, "Insulin action and resistance in obesity and type 2 diabetes," Nature Medicine, vol. 23, pp. 804-814, 2017.

[5] J. G. Burchfield, M. A. Kebede, C. C. Meoli et al., "High dietary fat and sucrose results in an extensive and time-dependent 
deterioration in health of multiple physiological systems in mice," The Journal of Biological Chemistry, vol. 293, no. 15, pp. 5731-5745, 2018.

[6] I. M. de Araujo, L. T. Parreiras-e-Silva, A. L. Carvalho, J. Elias, C. E. G. Salmon, and F. J. A. de Paula, "Insulin resistance negatively affects bone quality not quantity: the relationship between bone and adipose tissue," Osteoporosis International, vol. 31, no. 6, pp. 1125-1133, 2020.

[7] R. Ma, R. Zhu, L. Wang et al., "Diabetic osteoporosis: a review of its traditional Chinese medicinal use and clinical and preclinical research," Evidence-based Complementary and Alternative Medicine, vol. 2016, Article ID 3218313, 13 pages, 2016.

[8] V. V. Shanbhogue, D. M. Mitchell, C. J. Rosen, and M. L. Bouxsein, "Type 2 diabetes and the skeleton: new insights into sweet bones," The Lancet Diabetes and Endocrinology, vol. 4, pp. 159-173, 2016.

[9] N. Imerb, C. Thonusin, N. Chattipakorn, and S. C. Chattipakorn, "Aging, obese-insulin resistance, and bone remodeling," Mechanisms of Ageing and Development, vol. 191, article 111335, 2020.

[10] G. M. Pagnotti, M. Styner, G. Uzer et al., "Combating osteoporosis and obesity with exercise: leveraging cell mechanosensitivity," Nature Reviews. Endocrinology, vol. 15, no. 6, pp. 339-355, 2019.

[11] S. Rayalam, M. A. Della-Fera, and C. A. Baile, "Synergism between resveratrol and other phytochemicals: implications for obesity and osteoporosis," Molecular Nutrition \& Food Research, vol. 55, pp. 1177-1185, 2011.

[12] S. Khandelwal, "Obesity in midlife: lifestyle and dietary strategies," Climacteric, vol. 23, pp. 140-147, 2020.

[13] M. C. Oliveira, J. Vullings, and F. A. J. van de Loo, "Osteoporosis and osteoarthritis are two sides of the same coin paid for obesity," Nutrition, vol. 70, article 110486, 2020.

[14] K. Nowotny, T. Jung, A. Hohn, D. Weber, and T. Grune, "Advanced glycation end products and oxidative stress in type 2 diabetes mellitus," Biomolecules, vol. 5, pp. 194-222, 2015.

[15] H. Vlassara and J. Uribarri, "Advanced glycation end products (AGE) and diabetes: cause, effect, or both?," Current Diabetes Reports, vol. 14, p. 453, 2014.

[16] C. Liu, R. Zhu, H. Liu et al., "Aqueous extract of Mori folium exerts bone protective effect through regulation of calcium and redox homeostasis via PTH/VDR/CaBP and AGEs/ RAGE/Nox4/NF-kappaB signaling in diabetic rats," Frontiers in Pharmacology, vol. 9, p. 1239, 2018.

[17] M. Yamamoto and T. Sugimoto, "Advanced glycation end products, diabetes, and bone strength," Current Osteoporosis Reports, vol. 14, pp. 320-326, 2016.

[18] L. Wang, R. Ma, Y. Guo et al., “Antioxidant effect of Fructus Ligustri Lucidi aqueous extract in ovariectomized rats is mediated through Nox4-ROS-NF-kappaB pathway," Frontiers in Pharmacology, vol. 8, p. 266, 2017.

[19] S. Rathinavelu, C. Guidry-Elizondo, and J. Banu, "Molecular modulation of osteoblasts and osteoclasts in type 2 diabetes," Journal Diabetes Research, vol. 2018, article 6354787, 11 pages, 2018.

[20] K. H. Ding, Z. Z. Wang, M. W. Hamrick et al., "Disordered osteoclast formation in RAGE-deficient mouse establishes an essential role for RAGE in diabetes related bone loss," Biochemical and Biophysical Research Communications, vol. 340, pp. 1091-1097, 2006.
[21] R. Bucala, Z. Makita, G. Vega et al., "Modification of low density lipoprotein by advanced glycation end products contributes to the dyslipidemia of diabetes and renal insufficiency," Proceedings of the National Academy of Sciences of the United States of America, vol. 91, pp. 9441-9445, 1994.

[22] M. Grabowska, D. Wawrzyniak, K. Rolle et al., "Let food be your medicine: nutraceutical properties of lycopene," Food \& Function, vol. 10, pp. 3090-3102, 2019.

[23] Z. Yang, Z. Zhang, K. L. Penniston, N. Binkley, and S. A. Tanumihardjo, "Serum carotenoid concentrations in postmenopausal women from the United States with and without osteoporosis," International Journal for Vitamin and Nutrition Research, vol. 78, pp. 105-111, 2008.

[24] C. L. Shen, V. von Bergen, M.-C. Chyu et al., "Fruits and dietary phytochemicals in bone protection," Nutrition Research, vol. 32, no. 12, pp. 897-910, 2012.

[25] L. G. Rao, E. S. Mackinnon, R. G. Josse, T. M. Murray, A. Strauss, and A. V. Rao, "Lycopene consumption decreases oxidative stress and bone resorption markers in postmenopausal women," Osteoporosis International, vol. 18, pp. 109115, 2007.

[26] E. S. Mackinnon, A. V. Rao, R. G. Josse, and L. G. Rao, "Supplementation with the antioxidant lycopene significantly decreases oxidative stress parameters and the bone resorption marker N-telopeptide of type I collagen in postmenopausal women," Osteoporosis International, vol. 22, pp. 1091-1101, 2011.

[27] S. Fenni, H. Hammou, J. Astier et al., "Lycopene and tomato powder supplementation similarly inhibit high-fat diet induced obesity, inflammatory response, and associated metabolic disorders," Molecular Nutrition \& Food Research, vol. 61, no. 9, article 1601083, 2017.

[28] R. Zhu, B. Chen, Y. Bai et al., "Lycopene in protection against obesity and diabetes: a mechanistic review," Pharmacological Research, vol. 159, article 104966, 2020.

[29] R. Zhu, J. Wei, H. Liu et al., "Lycopene attenuates body weight gain through induction of browning via regulation of peroxisome proliferator-activated receptor $\gamma$ in high-fat dietinduced obese mice," The Journal of Nutritional Biochemistry, vol. 78, article 108335, 2020.

[30] R. Ma, L. Wang, B. Zhao et al., "Diabetes perturbs bone microarchitecture and bone strength through regulation of Sema3A/ IGF-1/ $\beta$-Catenin in rats," Cellular Physiology and Biochemistry, vol. 41, no. 1, pp. 55-66, 2017.

[31] L. Li, B. Chen, R. Zhu et al., "Fructus Ligustri Lucidi preserves bone quality through the regulation of gut microbiota diversity, oxidative stress, TMAO and Sirt6 levels in aging mice," Aging (Albany NY), vol. 11, no. 21, pp. 9348-9368, 2019.

[32] C. Bakici, R. O. Akgun, O. Ekim et al., "Three dimensional modeling and quantitative analysis of long bone parameters of rabbit using micro-computed tomography," Iranian Journal of Veterinary Research, vol. 22, no. 2, pp. 140-145, 2021.

[33] M. L. Bouxsein, S. K. Boyd, B. A. Christiansen, R. E. Guldberg, K. J. Jepsen, and R. Müller, "Guidelines for assessment of bone microstructure in rodents using micro-computed tomography," Journal of Bone and Mineral Research, vol. 25, no. 7, pp. 1468-1686, 2010.

[34] M. M. Tang, H. K. Tian, W. N. Yang et al., "Identification of constituents in Mori folium aqueous extract and drug serum," Chinese Journal of Experimental Traditional Medical Formulae, vol. 22, no. 9, pp. 25-29, 2016. 
[35] E. P. Paschalis, S. Gamsjaeger, D. N. Tatakis, N. Hassler, S. P. Robins, and K. Klaushofer, "Fourier transform infrared spectroscopic characterization of mineralizing type I collagen enzymatic trivalent cross-links," Calcified Tissue International, vol. 96, pp. 18-29, 2015.

[36] E. P. Paschalis, S. Gamsjaeger, and K. Klaushofer, "Vibrational spectroscopic techniques to assess bone quality," Osteoporosis International, vol. 28, pp. 2275-2291, 2017.

[37] E. A. Taylor, A. A. Lloyd, C. Salazar-Lara, and E. Donnelly, "Raman and Fourier transform infrared (FT-IR) mineral to matrix ratios correlate with physical chemical properties of model compounds and native bone tissue," Applied Spectroscopy, vol. 71, pp. 2404-2410, 2017.

[38] E. A. Taylor and E. Donnelly, "Raman and Fourier transform infrared imaging for characterization of bone material properties," Bone, vol. 139, article 115490, 2020.

[39] H. Ou-Yang, E. P. Paschalis, W. E. Mayo, A. L. Boskey, and R. Mendelsohn, "Infrared microscopic imaging of bone: spatial distribution of CO3(2-)," Journal of Bone and Mineral Research, vol. 16, no. 5, pp. 893-900, 2001.

[40] T. Sato, M. Iwaki, N. Shimogaito, X. Wu, S.-I. Yamagishi, and M. Takeuchi, "TAGE (toxic AGEs) theory in diabetic complications," Current Molecular Medicine, vol. 6, no. 3, pp. 351358, 2006.

[41] P. Sankar, B. Zachariah, V. Vickneshwaran, S. E. Jacob, and M. G. Sridhar, "Amelioration of oxidative stress and insulin resistance by soy isoflavones (from Glycine max) in ovariectomized Wistar rats fed with high fat diet: the molecular mechanisms," Experimental Gerontology, vol. 63, pp. 67-75, 2015.

[42] E. S. Ramos-Junior, G. A. Leite, C. C. Carmo-Silva et al., "Adipokine chemerin bridges metabolic dyslipidemia and alveolar bone loss in mice," Journal of Bone and Mineral Research, vol. 32, no. 5, pp. 974-984, 2017.

[43] H. L. Zhuang, X. Zhang, C. Zhu et al., "Molecular mechanisms of PPAR- $\gamma$; governing MSC osteogenic and adipogenic differentiation," Current Stem Cell Research \& Therapy, vol. 11, no. 3, pp. 255-264, 2016.

[44] P. K. Fazeli, M. C. Horowitz, O. A. MacDougald et al., "Marrow fat and bone-new perspectives," The Journal of Clinical Endocrinology and Metabolism, vol. 98, no. 3, pp. 935-945, 2013.

[45] J. Gautam, V. Khedgikar, P. Kushwaha et al., "Formononetin, an isoflavone, activates AMP-activated protein kinase/ $\beta$ catenin signalling to inhibit adipogenesis and rescues C57BL/ 6 mice from high-fat diet-induced obesity and bone loss," The British Journal of Nutrition, vol. 117, no. 5, pp. 645-661, 2017.

[46] M. Saito, Y. Kida, S. Kato, and K. Marumo, "Diabetes, collagen, and bone quality," Current Osteoporosis Reports, vol. 12, pp. 181-188, 2014.

[47] M. Saito and K. Marumo, "Bone quality in diabetes," Frontiers in Endocrinology, vol. 4, p. 72, 2013.

[48] M. M. Ardawi, M. H. Badawoud, S. M. Hassan et al., "Lycopene treatment against loss of bone mass, microarchitecture and strength in relation to regulatory mechanisms in a postmenopausal osteoporosis model," Bone, vol. 83, pp. 127-140, 2016.

[49] I. D. Figueiredo, T. F. O. Lima, M. D. Inácio et al., "Lycopene improves the metformin effects on glycemic control and decreases biomarkers of glycoxidative stress in diabetic rats," Diabetes, Metabolic Syndrome and Obesity: Targets and Therapy, vol. 13, pp. 3117-3135, 2020.
[50] N. Kumar Rajendran, B. P. George, R. Chandran, I. M. Tynga, N. Houreld, and H. Abrahamse, "The influence of light on reactive oxygen species and NF-кB in disease progression," Antioxidants (Basel), vol. 8, no. 12, p. 640, 2019.

[51] D. Zhao, X. Li, Y. Zhao et al., "Oleanolic acid exerts bone protective effects in ovariectomized mice by inhibiting osteoclastogenesis," Journal of Pharmacological Sciences, vol. 137, no. 1, pp. 76-85, 2018.

[52] D. T. Pierine, M. E. L. Navarro, I. O. Minatel et al., "Lycopene supplementation reduces TNF- $\alpha$ via RAGE in the kidney of obese rats," Nutrition \& Diabetes, vol. 4, no. 11, article e142, 2014.

[53] S. Tabrez, K. Z. Al-Shali, and S. Ahmad, "Lycopene powers the inhibition of glycation-induced diabetic nephropathy: a novel approach to halt the AGE-RAGE axis menace," BioFactors, vol. 41, pp. 372-381, 2015.

[54] Y. Jeong, J. Lim, and H. Kim, "Lycopene inhibits reactive oxygen species-mediated NF- $\kappa \mathrm{B}$ signaling and induces apoptosis in pancreatic cancer cells," Nutrients, vol. 11, no. 4, p. 762, 2019.

[55] E. S. Mackinnon, A. venket Rao, and L. G. Rao, "Dietary restriction of lycopene for a period of one month resulted in significantly increased biomarkers of oxidative stress and bone resorption in postmenopausal women," The Journal of Nutrition, Health \& Aging, vol. 15, no. 2, pp. 133-138, 2011.

[56] K. Li, G. Deng, Y. Deng et al., "High cholesterol inhibits tendon-related gene expressions in tendon-derived stem cells through reactive oxygen species-activated nuclear factor- $\kappa \mathrm{B}$ signaling," Journal of Cellular Physiology, vol. 234, no. 10, pp. 18017-18028, 2019.

[57] L. T. Ho-Pham, P. M. N. Chau, A. T. Do, H. C. Nguyen, and T. V. Nguyen, "Type 2 diabetes is associated with higher trabecular bone density but lower cortical bone density: the Vietnam osteoporosis study," Osteoporosis International, vol. 29, pp. 2059-2067, 2018.

[58] Y. Xu and Q. Wu, "Trends in osteoporosis and mean bone density among type 2 diabetes patients in the US from 2005 to 2014," Scientific Reports, vol. 11, p. 3693, 2021.

[59] M. Ebrahimpur, F. Sharifi, F. A. Nezhad et al., "Effect of diabetes on BMD and TBS values as determinants of bone health in the elderly: Bushehr elderly health program," Journal of Diabetes and Metabolic Disorders, vol. 18, pp. 99-106, 2019.

[60] P. Sihota, R. N. Yadav, R. Dhaliwal et al., "Investigation of mechanical, material, and compositional determinants of human trabecular bone quality in type 2 diabetes," The Journal of Clinical Endocrinology and Metabolism, vol. 106, no. 5, pp. e2271-e2289, 2021.

[61] S. Rokidi, V. F. C. Andrade, V. Borba et al., "Bone tissue material composition is compromised in premenopausal women with type 2 diabetes," Bone, vol. 141, article 115634, 2020.

[62] O. A. Nour, H. A. Ghoniem, M. A. Nader, and G. M. Suddek, "Impact of protocatechuic acid on high fat diet-induced metabolic syndrome sequelae in rats," European Journal of Pharmacology, vol. 907, article 174257, 2021.

[63] M. Aliabadi, F. Zamani-Garmsiri, G. Panahi, S. S. Tehrani, and R. Meshkani, "Metformin in combination with genistein ameliorates skeletal muscle inflammation in high-fat diet fed c57BL/6 mice," Cytokine, vol. 146, article 155638, 2021.

[64] S. Bornstein, M. Moschetta, Y. Kawano et al., "Metformin affects cortical bone mass and marrow adiposity in dietinduced obesity in male mice," Endocrinology, vol. 158, no. 10, pp. 3369-3385, 2017. 
[65] H. J. Son, J. Lee, S.-Y. Lee et al., "Metformin attenuates experimental autoimmune arthritis through reciprocal regulation of Th17/Treg balance and osteoclastogenesis," Mediators of Inflammation, vol. 2014, Article ID 973986, 13 pages, 2014.

[66] J. Lin, R. Xu, X. Shen, H. Jiang, and S. Du, "Metformin promotes the osseointegration of titanium implants under osteoporotic conditions by regulating BMSCs autophagy, and osteogenic differentiation," Biochemical and Biophysical Research Communications, vol. 531, pp. 228-235, 2020.

[67] T. Tankova, "Current indications for metformin therapy," Romanian Journal of Internal Medicine, vol. 41, pp. 215-225, 2003.

[68] L. Schurman, A. D. McCarthy, C. Sedlinsky et al., "Metformin reverts deleterious effects of advanced glycation end-products (AGEs) on osteoblastic cells," Experimental and Clinical Endocrinology \& Diabetes, vol. 116, no. 6, pp. 333-340, 2008.

[69] Y. Ishibashi, T. Matsui, M. Takeuchi, and S. Yamagishi, "Beneficial effects of metformin and irbesartan on advanced glycation end products (AGEs)-RAGE-induced proximal tubular cell injury," Pharmacological Research, vol. 65, pp. 297-302, 2012.

[70] L. Jiating, J. Buyun, and Z. Yinchang, "Role of metformin on osteoblast differentiation in type 2 diabetes," BioMed Research International, vol. 2019, Article ID 9203934, 6 pages, 2019.

[71] P. C. Marinello, C. Panis, T. N. X. Silva et al., "Oxidative stress and TGF- $\beta 1$ induction by metformin in MCF-7 and MDAMB-231 human breast cancer cells are accompanied with the downregulation of genes related to cell proliferation, invasion and metastasis," Pathology, Research and Practice, vol. 216, no. 10, article 153135, 2020.

[72] H. Y. Xu, W. Fang, Z. W. Huang et al., "Metformin reduces SATB2-mediated osteosarcoma stem cell-like phenotype and tumor growth via inhibition of N-cadherin/NF- $\kappa \mathrm{B}$ signaling," European Review for Medical and Pharmacological Sciences, vol. 21, no. 20, pp. 4516-4528, 2017.

[73] F. Bian, Y. Zhang, Y. L. Xie et al., "Effects of different concentrations of metformin on osteoclast differentiation and apoptosis and its mechanism," Pharmazie, vol. 76, no. 6, pp. 244-248, 2021. 